CLINICAL STUDY

\title{
Retinol-binding protein 4 is associated with insulin resistance, but appears unsuited for metabolic screening in women with polycystic ovary syndrome
}

\author{
Matthias Möhlig ${ }^{1,2}$, Martin O Weickert ${ }^{1,2}$, Elham Ghadamgahi ${ }^{1}$, Ayman M Arafat ${ }^{1}$, Joachim Spranger ${ }^{1,2}$, \\ Andreas F H Pfeiffer ${ }^{1,2}$ and Christof Schöfl ${ }^{1,3}$ \\ ${ }^{1}$ Department of Endocrinology, Diabetes and Nutrition, Charité-University Medicine Berlin, Campus Benjamin Franklin, 12200 Berlin, Germany, \\ ${ }^{2}$ Department of Clinical Nutrition, German Institute of Human Nutrition Potsdam-Rehbruecke, 14558 Nuthetal, Germany and ${ }^{3}$ Division of \\ Neuroendocrinology, Department of Neurosurgery, Friedrich-Alexander-University Erlangen-Nuremberg, Schwabachanlage 6, 91054 Erlangen, Germany \\ (Correspondence should be addressed to C Schöfl; Email: christof.schoefl@uk-erlangen.de)
}

\begin{abstract}
Objective: Adiposity, insulin resistance (IR), and hyperandrogenism are features of polycystic ovary syndrome (PCOS). Retinol-binding protein 4 (RBP4) secreted from adipose and liver tissues has been linked to IR. The impact of RBP4 on IR in PCOS and its usability to identify women with metabolic syndrome (MS) or impaired glucose tolerance ((IGT) or diabetes) were investigated.

Design: Plasma RBP4 was determined in 115 consecutive PCOS women. Associations with IR, body composition, and hyperandrogenemia were investigated by correlation and multiple linear regression analyses in 110 non-diabetics. Receiver operating characteristic curve analysis was used to evaluate RBP4 as a parameter for identifying MS and IGT or diabetes.

Results: RBP4 increased over tertiles of IR $(P=0.009)$. RBP4 correlated with HOMA \%S $(R=-0.286$, $P=0.002)$, waist-to-hip ratio (WHR) $(R=0.233, P=0.034)$, and dual energy X-ray absorptiometry (DEXA)-lean body mass $(R=0.282, P=0.016)$ but not with body mass index (BMI), DEXA-total or trunk fat mass, hsCRP, free testosterone, DHEAS, androstenedione, and $17 \beta$-estradiol. Adjusted for age, BMI, smoking, and IGT, the association between RBP4 and HOMA \%S remained significant $(P=0.032)$. RBP4 explained $4.6 \%$ of the variation in HOMA \%S. RBP4 was higher in MS and IGT or diabetes, but its ability to identify these women was low (area under the curve, $\mathrm{AUC}=0.631, P=0.041$ or $\mathrm{AUC}=0.660, P=0.016)$.

Conclusions: In PCOS, RBP4 has a small independent impact on IR. It is not correlated with hyperandrogenemia, $17 \beta$-estradiol, other adrenal steroids, or with markers of adiposity in general. Furthermore, RBP4 does not appear suitable for screening MS or impaired glucose metabolism (IGT or diabetes).
\end{abstract}

European Journal of Endocrinology 158 517-523

\section{Introduction}

Polycystic ovary syndrome (PCOS) is one of the most frequent endocrine diseases in women $(1,2)$, characterized by hyperandrogenism, chronic anovulation, and infertility. Many PCOS women are overweight or obese and suffer from insulin resistance (IR) and other features of metabolic syndrome (MS), which increase the risk for type 2 diabetes mellitus (DM) and cardiovascular disease (3-5). There is evidence that adiposity and associated metabolic alterations such as IR play an important role in the development and maintenance of PCOS pathology at least in a substantial proportion of patients (3-5). On the other hand, hyperandrogenemia may enhance IR and metabolic dysregulation seen in these patients (6). This could lead to a kind of vicious cycle that may explain the finding that women with PCOS are more insulin resistant than expected from age and body mass index (BMI) (7).

The pathophysiology that links adiposity, IR, and hyperandrogenism or chronic anovulation is only poorly understood. However, signals secreted from the adipose tissue are supposed to be involved (8). One of those factors is retinol-binding protein 4 (RBP4) that is secreted from the adipose tissue and the liver (9-12). In mice, overexpression of RBP4 has been shown to cause IR presumably by enhanced expression of the gluconeogenic enzyme phosphoenolpyruvate carboxykinase (PEPCK) and impairment of muscle insulin action (10). Therefore, RBP4 has been hypothesized to be a novel adipokine linking adiposity with systemic IR and potentially with adiposity-related disorders. In overweight women with PCOS compared with controls, enhanced expression of RBP4 in adipose tissue and 
elevated serum RBP4 has been recently described (13). Interestingly, RBP4 expression and secretion from adipose tissue was enhanced by $17 \beta$-estradiol. In another cohort, no differences in serum RBP4 were observed between lean PCOS women and BMI-matched controls (14). In that study, RBP4 correlated with BMI, body fat mass, and waist circumference, but not with indices of IR (14). This contrasts to yet another report that describes an association with IR as assessed by a euglycemic-hyperinsulinemic clamp as well as with the waist-to-hip ratio (WHR) but not with BMI (15). As with the relationship of RBP4 to IR, parameters of adiposity and gonadal steroids are far from clear, we addressed the potential role of RBP4 in IR in PCOS women and tested whether RBP4 might be a useful tool for identifying insulin-resistant women with MS or for identifying women with impaired glucose metabolism (impaired glucose tolerance (IGT) or DM), as suggested (16).

\section{Subjects and methods}

\section{Subjects}

One hundred and fifteen consecutive PCOS women who were referred to our clinic between 2001 and 2006 because of hirsutism or oligo/amenorrhea were included after written informed consent was obtained. The cohort has been published previously in part (17-20). The diagnosis of PCOS was based on: a) the presence of chronic ovulatory dysfunction, i.e., oligomenorrhea (four or less cycles in the last 6 months) or amenorrhea (no cycles in the last 6 months); b) clinical signs of hyperandrogenism, i.e., hirsutism as defined by a Ferriman-Gallwey Score $\geq 8$ (21); c) laboratory findings, i.e., hyperandrogenemia, defined as serum androgen levels (DHEAS, androstenedione, or total testosterone) above the upper limit of normal for the respective assay; and d) the exclusion of other disorders such as Cushing's syndrome, late-onset 21-hydroxylase deficiency, thyroid dysfunction, hyperprolactinemia, or androgen-secreting tumors. These diagnostic criteria for PCOS are consistent with the most commonly used diagnostic criteria for PCOS, often referred to as the NIH consensus criteria (22). Twelve patients were euthyroid under thyroid hormone replacement because of Hashimoto's thyroiditis. Six women had arterial hypertension and were normotensive under antihypertensive drugs. Five women suffered from asthma, allergic rhinitis, or hyperkinetic heart syndrome. One woman was under anti-epileptic medication. Ninety-one women were not taking any medication.

All women were studied within the first 10 days following menstruation in the case of mild oligomenorrhea or at random if they suffered severe oligo- or amenorrhea. Blood was sampled in the morning after an overnight fast, and the samples were stored at $-20{ }^{\circ} \mathrm{C}$ until analysis. An oral glucose tolerance test (OGTT) was performed in all women to define

glucose metabolism. Five patients were newly diagnosed as suffering from DM. If not stated otherwise the five women with DM according to the revised American Disease Association (ADA) criteria (23) were excluded from the analysis because of the endpoint IR. Among the remaining 110 women, 19 suffered from IGT. The clinical and endocrine features of these 110 women are given in Table 1. IR was quantified by calculating HOMA \%S using the mean of three fasting glucose and insulin values (24) and the HOMA2 program kindly provided by Dr Levy.

\section{Assessment of body composition}

BMI was calculated as body weight (kg) divided by body height squared $\left(\mathrm{m}^{2}\right)$. Body fat and lean body masses were assessed in a subgroup of 73 women using wholebody scans by dual energy X-ray absorptiometry (DEXA; Lunar, Madison, WI, USA). Coefficient of variance was determined by repeated measurements and was $2.2 \%$ for total fat mass and $1 \%$ for lean body mass.

\section{Assays}

All biochemical and endocrine parameters were determined as described previously (18). Free testosterone was calculated from total testosterone and sex hormone-binding globulin as published (25) using a web-based calculator (http://www.issam.ch/freetesto.htm). The CAG repeat length polymorphism within the androgen receptor was determined in a subgroup of 61 women as described (26) and the mean CAG length was used for further calculations. For the measurement of RBP4, plasma samples were thawed for the first time and RBP4 was determined in

Table 1 Clinical and endocrine features of the non-diabetic polycystic ovary syndrome (PCOS) cohort $(n=110)$.

\section{Characteristic}

Age (years)

BMI $\left(\mathrm{kg} / \mathrm{m}^{2}\right)$

Fasting plasma glucose $(\mathrm{mmol} / \mathrm{l})$

Fasting insulin (pmol/l)

HOMA \%S

hsCRP (mg/l)

Calculated free testosterone $(\mathrm{pmol} / \mathrm{l})$

Estradiol (pmol/l)

Progesterone $(\mathrm{nmol} / \mathrm{l})$

$\mathrm{LH}(\mathrm{U} / \mathrm{l})$

$\mathrm{LH} / \mathrm{FSH}$

DHEAS (nmol/l)

Androstenedione $(\mathrm{nmol} / \mathrm{l})$

SHBG (nmol/l)

17-OH-progesterone $(\mathrm{nmol} / \mathrm{l})$

DEXA-total fat mass $(\mathrm{kg})$

DEXA-lean body mass $(\mathrm{kg})$

Overweight/obese subjects $\left(\mathrm{BMI}>25 \mathrm{~kg} / \mathrm{m}^{2}\right)$

Smoker

Continuous variables are given as mean \pm S.E.M. and frequencies as $n(\%)$. 
duplicate using a commercially available sandwich ELISA (lot no. 0604-304; Immundiagnostik, Bensheim, Germany), which is distributed in the United States by ALPCO Diagnostics (Salem, NH, USA) The intra- and interassay coefficients of variation were 3.9 and $5.1 \%$ respectively. According to the manufacturer's instruction, the polyclonal antibody used was generated against urinary RBP4, but detects both urinary and full-length RBP4. Full-length RBP4 serves as the standard in the ELISA and RBP4 can be measured both in plasma and serum samples.

\section{Statistical analysis}

Statistical analyses were performed with SPSS software (version 14.0; SPSS Inc., Chicago, IL, USA). Values are reported as mean \pm S.E.M. Significance was considered at two-tailed $\alpha<0.05$. Correlation analyses were performed using the Spearman correlation coefficient. The non-diabetic women were divided into tertiles of HOMA \%S. RBP4 was In transformed to achieve normal distribution according to the Shapiro-Wilk test. Differences in In-RBP4 between tertiles were compared by ANOVA. Differences in ln-RBP4 between women with or without the MS; (according to the ATPIII definition (27)) and women with or without IGT or DM were compared by Student's $t$-test. The relation between RBP4 and further variables with respect to IR (HOMA $\%$ S) was addressed by multiple linear regression analysis. The quality of the model was derived from the $R^{2}$ value. The impact of RBP4 on the dependent variable was calculated as standardized $\beta \times$ correlation $\times 100$. Receiver operating characteristic $(\mathrm{ROC})$ curve analysis was used to define the power of RBP4 and HOMA $\%$ S to identify PCOS women with MS, IGT, or DM. ROC curves were compared for significant differences using MedCalc Software (MedCalc, Mariakerke, Belgium).

\section{Results}

The clinical and endocrine characteristics of the nondiabetic women are depicted in Table 1 . RBP4 ranged from 8.8 to $44.2 \mathrm{mg} / \mathrm{l}$ with a mean value of $21.99 \pm$ $0.637 \mathrm{mg} / \mathrm{l}(n=110)$.

\section{RBP4 is associated with IR in PCOS women}

To examine the association between RBP4 and IR, the study cohort was divided into tertiles of HOMA \%S. Tertile 1 comprised the most insulin-resistant women (HOMA \%S ranging from 13.6 to $49.2 \%$ S) and tertile 3 contained the most insulin-sensitive women (HOMA \%S ranging from 86.6 to $232 \% \mathrm{~S}$ ). The concentrations of RBP4 significantly increased with increasing IR as depicted in Fig. 1 (ln-RBP4 values were significantly different between the three groups, $P=0.009$, tertile 1 :

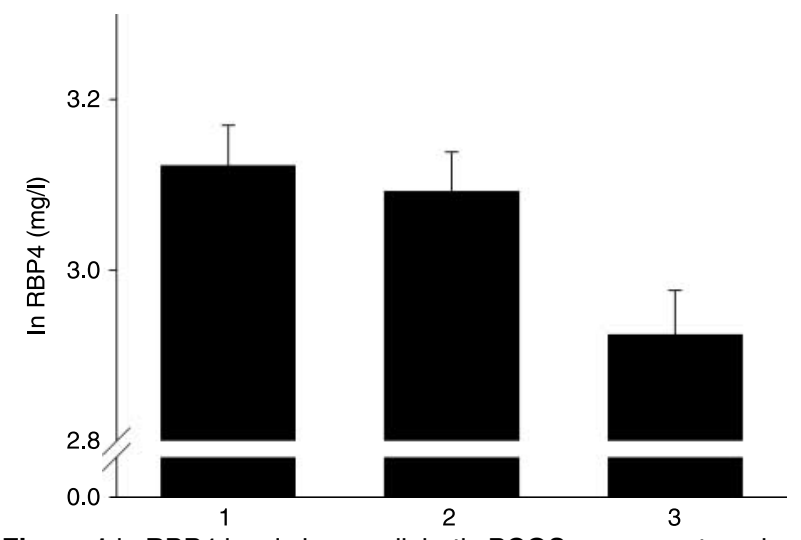

Figure 1 In RBP4 levels in non-diabetic PCOS women categorized by tertiles of HOMA \%S. Tertile 1 is the most insulin-resistant tertile. $P$ value for difference is 0.009 .

In-RBP4 3.12 $\pm 0.05 \mathrm{mg} / \mathrm{l}$, tertile 2: $\ln -\mathrm{RBP} 4$ $3.09 \pm 0.05 \mathrm{mg} / \mathrm{l}$, tertile $3: \ln -\mathrm{RBP} 42.92 \pm 0.05 \mathrm{mg} / \mathrm{l})$. Furthermore, there was a significant correlation between RBP4 and HOMA \%S $(R=-0.286$, $P=0.002, n=110$; Fig. 2). The association between RBP4 and HOMA \%S was still significant after adjustment for age, BMI, smoking, and impaired glucose tolerance (IGT) using linear regression analysis (Table 2). In this model, RBP4 explained $4.6 \%$ of the variation in HOMA \%S (Table 2). Further adjustment for parameters of hyperandrogenemia (free testosterone, DHEAS, and androstenedione; $\beta=-0.182, P=0.018$, $n=98$ ) and for drug use did not change the result.

The best model so far to explain the variability in HOMA \%S in our cohort included age, smoking, IGT, BMI, WHR, free testosterone, the androgen receptor CAG repeat polymorphism length, and its multiplicative interaction with free testosterone (26). This model explained $58.1 \%$ of the variation in HOMA \%S. The inclusion of RBP4 slightly improved that model which now explains $63.4 \%$ of the variation in HOMA \%S. The impact of RBP4 on HOMA \%S was calculated as $7.6 \%$ $(\beta=-0.242, P=0.009, n=61)$, while age, smoking, WHR, and IGT had no significant impact.

\section{In PCOS women, RBP4 is neither associated with parameters of adiposity in general nor with subclinical inflammation or hyperandrogenemia}

The associations between RBP4 and determinants of IR in PCOS women, such as parameters of adiposity and hyperandrogenemia, were tested. Plasma RBP4 significantly correlated with WHR $(R=0.233, P=0.034$, $n=83)$ and DEXA-lean body mass $(R=0.282, P=0.016$, $n=72)$, but not with BMI $(P=0.16, n=109)$, DEXA-total fat mass $(P=0.54, n=73)$, or DEXA-trunk fat mass $(P=0.50, n=73)$. The significance of the associations with WHR and DEXA-lean body mass were lost when the $P$ values were corrected for the five endpoints used to 


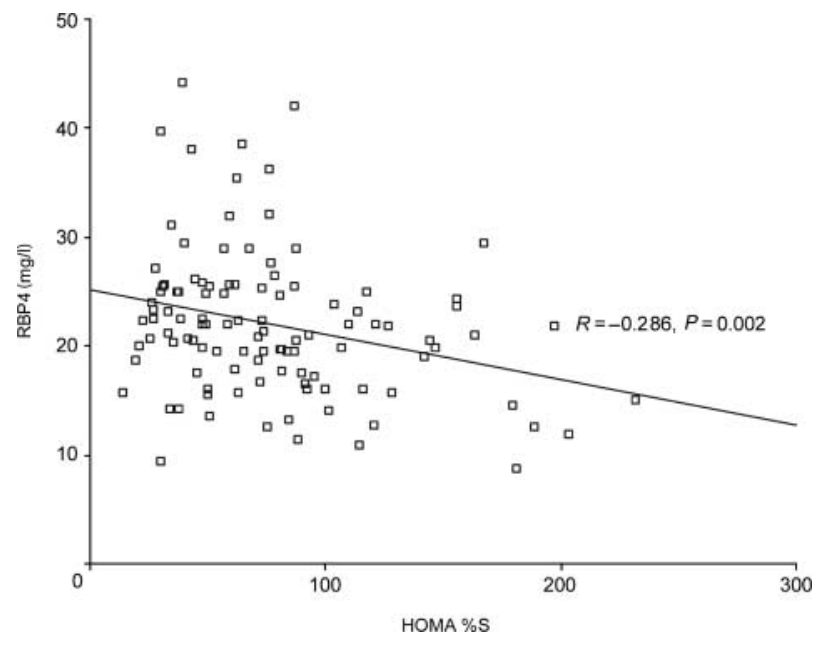

Figure 2 Correlation between RBP4 and HOMA \%S in non-diabetic PCOS women.

describe body composition according to Bonferroni. As subclinical inflammation is associated with IR and adiposity, the association of plasma RBP4 with hsCRP was tested, which was negative $(P=0.287, n=74)$. Furthermore, there was no correlation of RBP4 with free testosterone $(P=0.112, n=103)$ and other androgens or sex steroids such as DHEAS $(P=0.211, n=106)$, androstenedione $(P=0.581, n=108)$, or oestradiol $(P=0.770, n=107)$.

\section{Is RBP4 useful in identifying PCOS women with the MS or impaired glucose metabolism?}

RBP4 has been previously suggested as a potential marker to identify individuals with IR and a cardiovascular risk profile prior to the development of frank diabetes (16). We, therefore, tested whether RBP4 could be of value in identifying PCOS women suffering from the MS (defined according to Adult Treatment Panel III Criteria (27)) or from IGT or DM (23). Plasma RBP4 was elevated both in women with the MS (ln-RBP4 3.12 \pm $0.04 \mathrm{mg} / \mathrm{l}, n=29$ versus $\mathrm{ln}-\mathrm{RBP} 43.00 \pm 0.04, n=70, P$ value for difference: 0.027 ) and in women with IGT or DM ( $\ln -\mathrm{RBP} 43.18 \pm 0.05 \mathrm{mg} / \mathrm{l}, n=24$ versus $\ln -\mathrm{RBP} 4$ $3.02 \pm 0.03, n=91, P$ value for difference: 0.018 ) as depicted in Fig. 3. ROC curve analysis was used to analyze the suitability of plasma RBP4 to identify PCOS women with MS or with IGT or DM. The ROC curves for both endpoints (MS and IGT or DM) were significantly different from the bisecting line (MS: area under the curve (AUC) 0.631, $P=0.041$; IGT or DM: AUC 0.660, $P=0.016$ ). In our cohort, we have recently described that HOMA $\% \mathrm{~S}$ was the best-suited parameter to identify women with IGT or DM (28). We therefore compared RBP4 with HOMA \%S. Concerning the endpoint MS, the AUC of the ROC curve for HOMA \%S was significantly greater than the one for RBP4 (AUC HOMA \%S 0.87, $P<0.001$ for the difference to the bisecting line, $P<0.001$ for the pairwise comparison with the ROC curve for RBP4). For the endpoint IGT or DM, the ROC curve for HOMA \%S (AUC 0.74, P<0.001 for the difference to the bisecting line) was not significantly different from the ROC curve for RBP4 ( $P=0.31$ for the pairwise comparison). We further compared the suitability of RBP4 and HOMA \%S to predict IGT or DM by calculating the specificity at a given sensitivity of $95 \%$. The specificity was $31 \%$ for RBP 4 and $54 \%$ for HOMA $\%$ S at the $95 \%$ sensitivity cut point. Therefore, RBP4 compared with HOMA \%S appears less apt to identify PCOS women with IGT or DM. This is in line with the assumption that in general an AUC between 0.6 and 0.7 indicates a poor test. Therefore, RBP4 measurements in PCOS women do not appear to be clinically useful to identify women who are at risk for cardiovascular disease, a pre-diabetic state or early DM.

\section{Discussion}

Adiposity, IR, and hyperandrogenism are key features in many women with PCOS (3-5). IR associates with metabolic disturbances that render PCOS women to an increased risk for type 2 diabetes and cardiovascular disease $(29,30)$. However, the molecular mechanisms involved in this complex interplay between adiposity, IR, hyperandrogenemia, and diabetic and cardiovascular risk factors are only incompletely understood.

RBP4, which is secreted from adipose and liver tissues (9-12), has been linked to IR in both rodent and human studies $(10,16,31,32)$. In our non-diabetic PCOS women who ranged from very insulin sensitive (HOMA $\%$ S 232.0\%) to highly insulin resistant (HOMA \%S $13.6 \%)$, RBP4 was significantly associated with HOMA $\% \mathrm{~S}$, a well-introduced index of IR, which is derived from

Table 2 Multiple linear regression analysis with HOMA \%S as the dependent variable $\left(R^{2}=0.478\right)$. Impact of each significantly associated variable on $\mathrm{HOMA} \% \mathrm{~S}$ as dedicated by the explanation of the variation in HOMA \%S (calculated as standardized $\beta$ coefficient $\times$ correlation $\times 100)$.

\begin{tabular}{lccccc}
\hline & RBP4 $(\mathrm{mg} / \mathrm{l})$ & BMI $\left(\mathrm{kg} / \mathrm{m}^{2}\right)$ & Age $($ years $)$ & IGT (no/yes) & Smoking (no/yes) \\
\hline Standardized $\beta$ coefficient & $-0.161(0.032)$ & $-0.547(<0.001)$ & $0.274(<0.001)$ & $-0.205(0.006)$ & $-0.039(0.59)$ \\
$(P$ value $)$ & -0.288 & -0.568 & 0.203 & -0.291 & -0.131 \\
Correlation & 4.64 & 31.07 & 5.56 & 5.92 & \\
Impact on HOMA \%S $(\%)$ & & & & \\
\hline
\end{tabular}



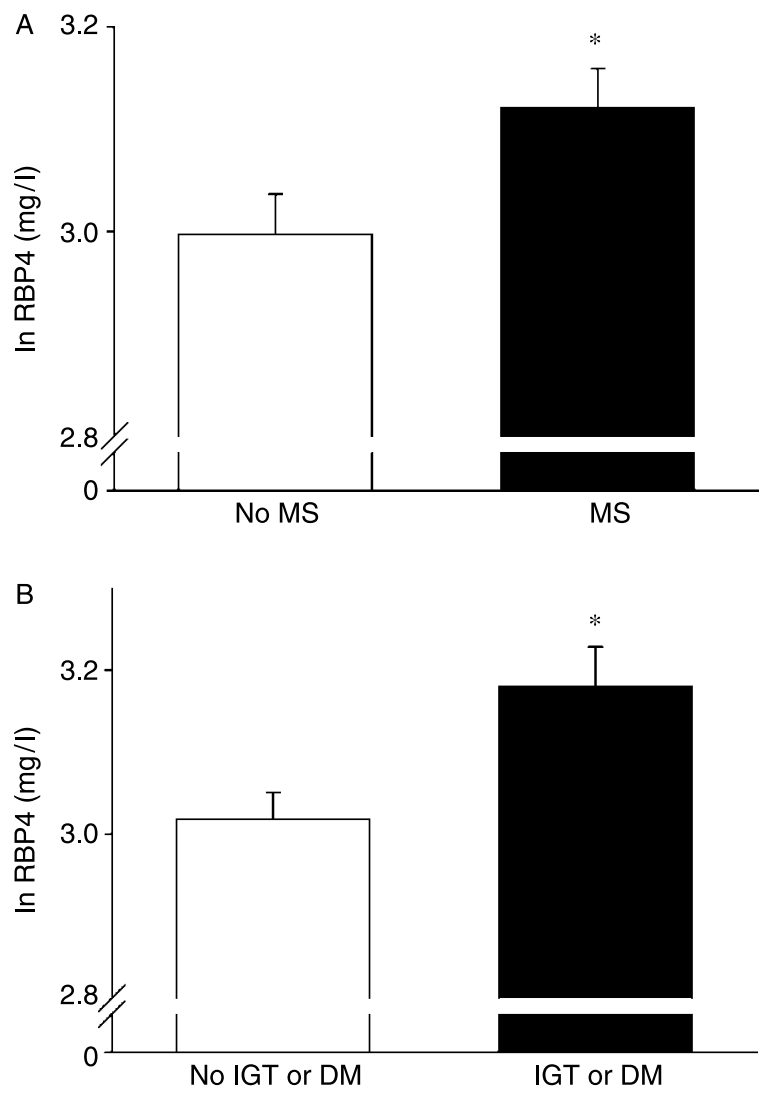

Figure 3 (A) In RBP4 levels in PCOS women with or without metabolic syndrome, $P$ value for difference is 0.027 . (B) In RBP4 levels in PCOS women with or without IGT or DM, $P$ value for difference is 0.018 .

parameters of the fasting state. The relationship between RBP4 and HOMA \%S was still significant even after adjustment for BMI, age, smoking, and IGT. RBP4, however, explained only $4.6 \%$ of the variation in HOMA $\%$ S. In a multiple regression model, that included age, smoking, BMI, WHR, IGT, free testosterone, and the androgen receptor $\mathrm{CAG}$ repeat polymorphism length and its multiplicative interaction with free testosterone, RBP4 also had an independent impact on IR leading to further improvement of the model. Again, the impact of $\mathrm{RBP} 4$ on $\mathrm{HOMA} \% \mathrm{~S}$ was relatively small explaining $7.6 \%$ of the variation in HOMA \%S. Our finding is well in line with another study in PCOS women who showed an inverse relation between RBP4 and $M$ value obtained during a euglycemic clamp study (15). HOMA \%S correlates well with clamp data and has been introduced as a useful tool to assess IR in larger populations (24). However, to fully exploit its potential, the calculation of HOMA \%S should be based on the mean of three fasting values for glucose and insulin and should be restricted to non-diabetic persons (24). These measures were recommended to minimize erroneous results caused by insulin pulsatility or advanced $\beta$-cell failure in DM (24). Thus, the relatively small impact of RBP4 on IR as described here, together with differences in the assessment of IR, may offer an explanation why the associations between RBP4 and IR in PCOS women as well as in other subjects reported so far are controversial (13-15, 31-34). Furthermore, cohort size, selection bias, or methodological differences in RBP4 measurements could contribute to obscure this relatively small impact on IR observed. Recently, a comparison of different methods and assays to measure RBP4 in blood has led to the suggestion that RBP4 should be preferentially determined by quantitative Western blotting in serum samples (35). Whether the measurement of RBP4 in serum by Western blotting is really superior and leads to different results and conclusions needs further evaluation. A recent paper by Yao-Borengasser et al. (34), in which RBP4 was measured in plasma and serum by the method used here, and by Western blotting, reported the same findings irrespective of the method of RBP4 measurement used. Furthermore, microalbuminuria has been described as a confounding factor that appears to increase RBP4-levels in diabetic patients (36). Since our analyses were restricted to non-diabetic patients significant distortion of the results by undiagnosed diabetic nephropathy appears unlikely.

In our PCOS patients with a range of BMI from underweight $\left(18 \mathrm{~kg} / \mathrm{m}^{2}\right)$ to severe obesity $\left(52 \mathrm{~kg} / \mathrm{m}^{2}\right)$, the WHR and DEXA-lean body mass were significantly associated with RBP4, whereas other parameters of adiposity or body composition like BMI, DEXA-total, or -trunk fat mass were not. The published data on the relation of RBP4 levels with various markers of obesity are inconsistent $(9,10,16,31,32,34,37-40)$, which fits well to the result seen here showing that RBP4 is correlated with some but not all parameters of adiposity. In our study, the significance of the associations between RBP4 and WHR or DEXA-lean body mass were lost after correction for testing multiple endpoints. It therefore appears that there is only a weak (if any) significant link between circulating RBP4 and the degree of general adiposity in our cohort. Besides adipose tissue $(11,40)$, the liver is the second major source for RBP4 $(12,41)$ and data were published demonstrating an association of liver fat content with RBP4, rather than with other fat depots (32). Therefore, the possibility exists that the variable associations between RBP4 and markers of adiposity observed by us and others reflect the more or less stringent correlation between liver fat content and BMI or other markers of body composition in the respective cohorts.

RBP4 is secreted from hepatocytes into the blood stream bound to transthyretin $(41,42)$. Transthyretin is a negative acute phase protein that decreases in acute inflammation (43). Therefore, RBP4 may decrease in inflammatory states. On the other hand, enhanced inflammation in accumulating adipose tissue has been shown to correlate both with increased RBP4 expression from s.c. adipose tissue and with elevated plasma RBP4 
(34). In our cohort of PCOS women, subclinical inflammation strongly associates with obesity and IR (18). We tested for an association between RBP4 and hsCRP as a central inflammatory marker. No correlation, however, could be delineated, which is line with a recent report from children (37). The data, however, reported from children are disputed (44). In PCOS women, we have no evidence for an association between RBP4 and inflammatory processes.

Very recently estradiol has been discussed as a putative regulator of $\mathrm{RBP} 4$, as its expression from human adipose tissue can be stimulated by $17 \beta$ estradiol (13). Since relative hyperoestrogenemia as well as elevated androgen levels are key features of many PCOS patients, we investigated the relationship between sex steroids and RBP4 levels. We found, however, no associations between plasma RBP4 and systemic gonadal or adrenal steroids. This is in accordance with other studies in PCOS $(14,15)$ and with the finding that plasma RBP4 is not elevated in PCOS women per se (14), but contrasts to another report based on a small group of ten obese PCOS and control women, which described a very close relation between RBP4 and estradiol, testosterone, DHEAS, and androstenedione (13).

It has been reported that RBP4 is elevated even before the development of frank diabetes and appears to identify IR and associated cardiovascular risk factors in subjects with varied clinical presentations (16). Consistently, we found RBP4 levels higher in women suffering from MS or impaired glucose metabolism (IGT or DM) as has been reported previously from PCOS and other cohorts $(14,31,45)$. ROC curve analysis, however, revealed that plasma RBP4 as determined here does not appear to be a clinically useful parameter to identify women suffering from either the MS, the IGT, or the DM. For both endpoints, the measurement of RBP4 was inferior to the calculation of HOMA \%S.

Taken together, plasma RBP4 has a small, but independent, impact on IR in PCOS. RBP4 is neither correlated with hyperandrogenemia, 17 $\beta$-estradiol, other adrenal steroids, nor in general with markers of obesity. Despite RBP4 levels being increased in PCOS women with MS or impaired glucose metabolism (IGT or DM), RBP4 appears unsuited for the clinical screening of PCOS women for metabolic alterations such as MS or IGT and DM.

\section{Acknowledgements}

The authors thank B Faust and K Sprengel for their excellent technical assistance. There are no sources of external funding to declare. The study used departmental research funds allocated by the Charité and no conflict of interest exists.

\section{References}

1 Asuncion M, Calvo RM, San Millan JL, Sancho J, Avila S \& EscobarMorreale HF. A prospective study of the prevalence of the polycystic ovary syndrome in unselected Caucasian women from Spain. Journal of Clinical Endocrinology and Metabolism 200085 2434-2438.

2 Azziz R, Woods KS, Reyna R, Key TJ, Knochenhauer ES \& Yildiz BO. The prevalence and features of the polycystic ovary syndrome in an unselected population. Journal of Clinical Endocrinology and Metabolism 200489 2745-2749.

3 Franks S. Polycystic ovary syndrome. New England Journal of Medicine 1995333 853-861.

4 Dunaif A. Insulin resistance and the polycystic ovary syndrome: mechanism and implications for pathogenesis. Endocrine Reviews 199718 774-800.

5 Barber TM, McCarthy MI, Wass JA \& Franks S. Obesity and polycystic ovary syndrome. Clinical Endocrinology 200665 137-145.

6 Moghetti P, Tosi F, Castello R, Magnani CM, Negri C, Brun E, Furlani L, Caputo M \& Muggeo M. The insulin resistance in women with hyperandrogenism is partially reversed by antiandrogen treatment: evidence that androgens impair insulin action in women. Journal of Clinical Endocrinology and Metabolism 199681 952-960.

7 Dunaif A, Segal KR, Futterweit W \& Dobrjansky A. Profound peripheral insulin resistance, independent of obesity, in polycystic ovary syndrome. Diabetes 198938 1165-1174.

8 Staiger $\mathrm{H}$ \& Häring HU. Adipocytokines: fat-derived humoral mediators of metabolic homeostasis. Experimental and Clinical Endocrinology and Diabetes 2005113 67-79.

9 Janke J, Engeli S, Boschmann M, Adams F, Bohnke J, Luft FC, Sharma AM \& Jordan J. Retinol-binding protein 4 in human obesity. Diabetes $2006552805-2810$.

10 Yang Q, Graham TE, Mody N, Preitner F, Peroni OD, Zabolotny JM, Kotani K, Quadro L \& Kahn BB. Serum retinol binding protein 4 contributes to insulin resistance in obesity and type 2 diabetes. Nature $2005436356-362$.

11 Tsutsumi C, Okuno M, Tannous L, Piantedosi R, Allan M, Goodman DS \& Blaner WS. Retinoids and retinoid-binding protein expression in rat adipocytes. Journal of Biological Chemistry 1992 267 1805-1810.

12 Soprano DR, Soprano KJ \& Goodman DS. Retinol-binding protein messenger RNA levels in the liver and in extrahepatic tissues of the rat. Journal of Lipid Research 198627 166-171.

13 Tan BK, Chen J, Lehnert H, Kennedy R \& Randeva HS. Raised serum, adipocyte, and adipose tissue retinol-binding protein 4 in overweight women with polycystic ovary syndrome: effects of gonadal and adrenal steroids. Journal of Clinical Endocrinology and Metabolism 200792 2764-2772 (Epub 2007 Apr 2724).

14 Hahn S, Backhaus M, Broecker-Preuss M, Tan S, Dietz T, Kimmig R, Schmidt M, Mann K \& Janssen OE. Retinol-binding protein 4 levels are elevated in polycystic ovary syndrome women with obesity and impaired glucose metabolism. European Journal of Endocrinology 2007157 201-207.

15 Weiping L, Qingfeng C, Shikun M, Xiurong L, Hua Q, Xiaoshu B, Suhua Z \& Qifu L. Elevated serum RBP4 is associated with insulin resistance in women with polycystic ovary syndrome. Endocrine $200630283-287$.

16 Graham TE, Yang Q, Blüher M, Hammarstedt A, Ciaraldi TP, Henry RR, Wason CJ, Oberbach A, Jansson PA, Smith U \& Kahn BB. Retinol-binding protein 4 and insulin resistance in lean, obese, and diabetic subjects. New England Journal of Medicine 2006 $3542552-2563$.

17 Schöfl C, Horn R, Schill T, Schlösser HW, Müller MJ \& Brabant G. Circulating ghrelin levels in patients with polycystic ovary syndrome. Journal of Clinical Endocrinology and Metabolism 2002 $874607-4610$.

18 Möhlig M, Spranger J, Osterhoff M, Ristow M, Pfeiffer AF, Schill T, Schlösser HW, Brabant G \& Schöfl C. The polycystic ovary syndrome per se is not associated with increased chronic inflammation. European Journal of Endocrinology 2004150 525-532. 
19 Möhlig M, Flöter A, Spranger J, Weickert MO, Schill T, Schlösser HW, Brabant G, Pfeiffer AF, Selbig J \& Schöfl C. Predicting impaired glucose metabolism in women with polycystic ovary syndrome by decision tree modelling. Diabetologia $2006 \mathbf{4 9}$ 2572-2579.

20 Möhlig M, Weickert MO, Ghadamgadai E, Machlitt A, Pfüller B, Arafat AM, Pfeiffer AF \& Schöfl C. Adipocyte fatty acid-binding protein is associated with markers of obesity, but is an unlikely link between obesity, insulin resistance, and hyperandrogenism in polycystic ovary syndrome women. European Journal of Endocrinology 2007157 195-200.

21 Ferriman D \& Gallwey JD. Clinical assessment of body hair growth in women. Journal of Clinical Endocrinology and Metabolism 1961 21 1440-1447.

22 Zawadzki JK \& Dunaif A. Diagnostic criteria for polycystic ovary syndrome: towards a rational approach. In Current Issues in Endocrinology and Metabolism: Polycystic Ovary Syndrome, pp $377-$ 384. Eds A Dunaif, J Givens, F Haseltine \& G Merriam, New York: Blackwell, 1992.

23 American Diabetes Association. Clinical practice recommendations 2002. Diabetes Care 200225 S1-S147.

24 Matthews DR, Hosker JP, Rudenski AS, Naylor BA, Treacher DF \& Turner RC. Homeostasis model assessment: insulin resistance and beta-cell function from fasting plasma glucose and insulin concentrations in man. Diabetologia 198528 412-419.

25 Vermeulen A, Verdonck L \& Kaufman JM. A critical evaluation of simple methods for the estimation of free testosterone in serum. Journal of Clinical Endocrinology and Metabolism $1999 \mathbf{8 4}$ 3666-3672.

26 Möhlig M, Jürgens A, Spranger J, Hoffmann K, Weickert MO, Schlösser HW, Schill T, Brabant G, Schüring A, Pfeiffer AF, Gromoll J \& Schöfl C. The androgen receptor CAG repeat modifies the impact of testosterone on insulin resistance in women with polycystic ovary syndrome. European Journal of Endocrinology 2006 155 127-130.

27 National Institutes of Health: Third Report of the National Cholesterol Education Program Expert Panel on Detection, Evaluation, and Treatment of High Blood Cholesterol in Adults (Adult Treatment Panel III). NIH Publication 2001 01-3670.

28 Möhlig M, Spranger J, Ristow M, Pfeiffer AF, Schill T, Schlösser HW, Moltz L, Brabant G \& Schöfl C. Predictors of abnormal glucose metabolism in women with polycystic ovary syndrome. European Journal of Endocrinology $2006 \mathbf{1 5 4}$ 295-301.

29 Dahlgren E, Janson PO, Johansson S, Lapidus L \& Oden A. Polycystic ovary syndrome and risk for myocardial infarction. Evaluated from a risk factor model based on a prospective population study of women. Acta Obstetricia et Gynecologica Scandinavica 199271 599-604.

30 Ehrmann DA, Barnes RB, Rosenfield RL, Cavaghan MK \& Imperial J. Prevalence of impaired glucose tolerance and diabetes in women with polycystic ovary syndrome. Diabetes Care 199922 141-146.

31 Cho YM, Youn BS, Lee H, Lee N, Min SS, Kwak SH, Lee HK \& Park KS. Plasma retinol-binding protein-4 concentrations are elevated in human subjects with impaired glucose tolerance and type 2 diabetes. Diabetes Care 200629 2457-2461.

32 Stefan N, Hennige AM, Staiger H, Machann J, Schick F, Schleicher E, Fritsche A \& Häring HU. High circulating retinolbinding protein 4 is associated with elevated liver fat but not with total, subcutaneous, visceral, or intramyocellular fat in humans. Diabetes Care 200730 1173-1178.

33 von Eynatten M, Lepper PM, Liu D, Lang K, Baumann M, Nawroth PP, Bierhaus A, Dugi KA, Heemann U, Allolio B \&
Humpert PM. Retinol-binding protein 4 is associated with components of the metabolic syndrome, but not with insulin resistance, in men with type 2 diabetes or coronary artery disease. Diabetologia $2007 \mathbf{5 0}$ 1930-1937.

34 Yao-Borengasser A, Varma V, Bodles AM, Rasouli N, Phanavanh B, Lee MJ, Starks T, Kern LM, Spencer HJ, III, Rashidi AA, McGehee RE Jr, Fried SK \& Kern PA. Retinol binding protein 4 expression in humans: relationship to insulin resistance, inflammation, and response to pioglitazone. Journal of Clinical Endocrinology and Metabolism 200792 2590-2597.

35 Graham TE, Wason CJ, Bluher M \& Kahn BB. Shortcomings in methodology complicate measurements of serum retinol binding protein (RBP4) in insulin-resistant human subjects. Diabetologia $2007 \mathbf{5 0} 814-823$.

36 Raila J, Henze A, Spranger J, Möhlig M, Pfeiffer AF \& Schweigert FJ. Microalbuminuria is a major determinant of elevated plasma retinol-binding protein 4 in type 2 diabetic patients. Kidney International $2007 \mathbf{7 2}$ 505-511.

37 Aeberli I, Biebinger R, Lehmann R, L’Allemand D, Spinas GA \& Zimmermann MB. Serum retinol-binding protein 4 concentration and its ratio to serum retinol are associated with obesity and metabolic syndrome components in children. Journal of Clinical Endocrinology and Metabolism $2007 \mathbf{2 8} 28$.

38 Broch M, Vendrell J, Ricart W, Richart C \& Fernandez-Real JM. Circulating retinol-binding protein-4, insulin sensitivity, insulin secretion, and insulin disposition index in obese and nonobese subjects. Diabetes Care 200730 1802-1806.

39 Gavi S, Stuart LM, Kelly P, Melendez MM, Mynarcik DC, Gelato MC \& McNurlan MA. Retinol-binding protein 4 is associated with insulin resistance and body fat distribution in nonobese subjects without type 2 diabetes. Journal of Clinical Endocrinology and Metabolism 200792 1886-1890.

40 Klöting N, Graham TE, Berndt J, Kralisch S, Kovacs P, Wason CJ, Fasshauer M, Schon MR, Stumvoll M, Bluher M \& Kahn BB. Serum retinol-binding protein is more highly expressed in visceral than in subcutaneous adipose tissue and is a marker of intra-abdominal fat mass. Cell Metabolism 20076 79-87.

41 Bellovino D, Morimoto T, Tosetti F \& Gaetani S. Retinol binding protein and transthyretin are secreted as a complex formed in the endoplasmic reticulum in HepG2 human hepatocarcinoma cells. Experimental Cell Research 1996222 77-83.

42 Blaner WS. Retinol-binding protein: the serum transport protein for vitamin A. Endocrine Reviews 198910 308-316.

43 Gabay C \& Kushner I. Acute-phase proteins and other systemic responses to inflammation. New England Journal of Medicine 1999 340 448-454.

44 Balagopal P, Graham TE, Kahn BB, Altomare A, Funanage V \& George D. Reduction of elevated serum retinol binding protein in obese children by lifestyle intervention: association with subclinical inflammation. Journal of Clinical Endocrinology and Metabolism 200792 1971-1974.

45 Qi Q, Yu Z, Ye X, Zhao F, Huang P, Hu FB, Franco OH, Wang J, Li H, Liu Y \& Lin X. Elevated retinol-binding protein 4 levels are associated with metabolic syndrome in Chinese people. Journal of Clinical Endocrinology and Metabolism 20071818.

Received 21 December 2007

Accepted 3 January 2008 